\section{Einladung zur ordentlichen Mitgliederversammlung am 29. April 2022}

Liebe Kolleginnen und Kollegen,

die vergangenen zwei Jahre der berufspolitischen Arbeit im BDRh waren geprägt von der Pandemie. Auch wenn vieles nur virtuell möglich war, war es für den BDRh eine sehr aktive und - so hoffen wir - auch erfolgreiche Zeit. Doch zu kurz kamen der Austausch, das Kreative und die Gespräche mit Mitgliedern. Daher haben wir uns entschieden, den nächsten Jahreskongress und die damit verbundene Mitgliederversammlung in Präsenz durchzuführen - natürlich unter Beachtung der dann geltenden Maßnahmen. Wir laden Sie daher herzlich ein zur Ordentlichen Mitgliederversammlung des BDRh am Freitag, 29. April 2022 im Anschluss an den BDRhKongress, von 18:30 bis 19.45 Uhr im Seminaris CampusHotel, Takustr. 39, 14195 Berlin-Dahlem.

Im Anhang finden Sie die vorläufige Tagesordnung. Bitte beachten Sie, dass die Mitgliederversammlung wie auch der gesamte Kongress unter 2G-Bedingungen stattfinden wird.

Bei Fragen wenden Sie sich gerne an unsere Geschäftsführerin Sonja Froschauer.

Mit besten Grüßen

\section{Dr. Silke Zinke}

1. Vorsitzende des Vorstands

\section{BERUFSVERBAND}

BDRh

DEUTSCHER

RHEUMATOLOGEN E.V.

Berufsverband Deutscher Rheumatologen (BDRh)

Dr. Silke Zinke (V.i.S. d.P.)

1. Vorsitzende

Dr.-Max-Str. 21

82031 Grünwald

Tel: 089 - 90414141 0, Fax. 089 - 904141419

\title{
Vorläufige Agenda
}

TOP

1 Bericht des Vorstandes zu aktuellen Themen und Projekten (u.a. Versorgungsatlas)

2 Bericht des Kassenwarts

3 Aussprache zu aktuellen Themen

4 Satzungsänderung: Ermöglichung von Mitgliederversammlungen auf dem Wege der Videokonferenz

5 Verschiedenes 